\title{
The reliability of sampling three to six nodes for staging breast cancer
}

\author{
Gábor Cserni
}

\begin{abstract}
Aims-To test the hypothesis that a correct qualitative assessment of axillary nodal status can be established by examining only a limited number of lymph nodes.

Methods-Slides from 499 pN1 or pN0 axillary dissection specimens relating to symptomatic breast cancer cases operated on at our institution between 1991 and 1996 were reviewed. Nodes were ranked in descending order on the basis of their estimated size and lymphoid or metastatic tissue content. After ranking, all nodes were studied microscopically; 265 axillary clearance specimens were positive.

Results-Assessment of the 3-6 largest/ firmest nodes can lead to the detection of 93-98\% of node positive patients and can give a correct qualitative assessment of axillary node status in $96-99 \%$.

Conclusions-Sampling the 4-6 largest/ firmest nodes seems to be a reliable alternative for the staging of symptomatic breast cancer. These results suggest a reconsideration of the generally held view that a minimum of 10 nodes is required for adequate identification of the $\mathrm{pN} 0$ category.

(F Clin Pathol 1999;52:681-683)
\end{abstract}

Keywords: axillary clearance; axillary sampling; breast cancer

The axillary lymph node status is still the most important single prognostic factor in breast cancer. Clinical examination ${ }^{12}$ and the use of imaging techniques ${ }^{3-5}$ to assess the node status are not reliable enough procedures because of the high rates of false positive and false negative results. Histopathological assessment has rather been considered the gold standard for the evaluation of lymph nodes. However, there are numerous ways to assess the axillary lymph nodes, depending on the surgical procedure performed and the histopathological tools used. Complete axillary dissection and the routine histopathological work up of a minimum of 10 to 11 nodes was once the standard staging and therapeutic procedure, but several alternative ways have been studied to reduce possible complications of the dissection and to spare the increasing proportion of patients without axillary metastases. Axillary sampling $^{6-10}$ and sentinel lymph node biopsy ${ }^{1-14}$ are suitable alternatives, with the aim of staging the disease and identifying the need for further treatment of the axilla. Accumulating data suggest that sentinel node assessment will play the major role in the future of axillary surgery for a considerable number of patients, but this does not fall in the scope of the present paper. On the other hand, there is controversy over axillary sampling as a means of evaluating the axilla; it is not a uniform procedure and has many opponents. ${ }^{15-20}$

I have used our archive of histopathology reports and slides to assess the value of examining a limited number of lymph nodes, modelling axillary sampling versus axillary dissection and examination of the full lymph node content.

\section{Methods}

Histopathology reports from the archives of department of pathology, Bács-Kiskun County Teaching Hospital, were reviewed and records relating to breast cancer cases treated by mastectomy or breast conserving surgery and axillary dissection between 1991 and 1996 were retrieved. Cases with categories $\mathrm{pN} 0$ or $\mathrm{pN} 1$ of the UICC TNM system ${ }^{21}$ were selected, and all slides of the axillary lymph nodes were reviewed.

Originally, lymph nodes were recovered from the axillary fat without clearing and were processed separately. In all cases, the largest cut surface was sectioned and studied under the microscope after haematoxylin and eosin (H\&E) staining.

In the present study, lymph nodes were numbered consecutively in sequence of size, the first being the largest. Lymphoid and metastatic neoplastic tissue content was also considered on the basis of the blue area (more nuclear staining) seen macroscopically on the H\&E stained slides. In this way, the largest node was not necessarily the one with the largest diameter, but the one with the largest estimated blue area-corresponding to either lymphoid or metastatic neoplastic tissue, or both. Nodes with similar estimated blue areas received the same sequential numbers (for example, three nodes each numbered $2-4$ would indicate that these three nodes were the $2 \mathrm{nd}, 3 \mathrm{rd}$, and 4 th largest ones, but that their estimated sizes were too similar for a distinction to be made between them). Nodes were then studied microscopically for the identification of metastases. The serial numbers of metastatic nodes were recorded.

The results of assessing only the first three, four, five, or six largest nodes were finally compared with the results of assessing all the lymph nodes.

\section{Results}

The effectiveness of assessing the first three, four, five, or six largest/firmest nodes against assessing all axillary nodes was tested. In all, 
Table 1 Rates of detection of positive nodes when only the first three, four, five, or six largest/firmest nodes were examined and the accuracy of this approach, expressed as the percentages of cases adequately identified, in comparison with the results of an assessment of the total number of nodes in the clearance specimens

\begin{tabular}{|c|c|c|c|c|}
\hline & \multicolumn{4}{|c|}{ Number of lymph nodes assessed } \\
\hline & 3 Nodes & 4 Nodes & 5 Nodes & 6 Nodes \\
\hline Positive axillary nodes & 236 to 246 & 250 to 257 & 253 to 259 & 258 to 261 \\
\hline $\begin{array}{l}\text { Per cent of group A } \\
\quad \text { (positive nodes only; } n=265 \text { ) } \\
\text { Per cent of group B }\end{array}$ & $89-93 \%$ & $94-97 \%$ & $95-98 \%$ & $97-98 \%$ \\
\hline (all nodes; $\mathrm{n}=499$ ) & $94-96 \%$ & $97-98 \%$ & $98-99 \%$ & $99 \%$ \\
\hline
\end{tabular}

265 axillary clearance specimens were positive (set A) from the 499 involved in the study (set B). Rates of concordance with the qualitative axillary nodal status established from the samples of the three to six largest nodes were expressed as percentages of the node positive cases (A) and of all the specimens (B) in table 1. As identical serial numbers were assigned to nodes of the same estimated size, the number of cases matching the qualitative axillary nodal status (positive or negative) was given as a range (for example, metastasis in only one of the two nodes $3-4$ could either be identified by assessing three nodes or be missed).

The mean number of lymph nodes per axillary specimen in this study was 10.7 (range 1-45), while the mean number of metastatic nodes was 2.5 (range $0-43$ ).

\section{Discussion}

With this system of serial numbering the lymph nodes on the basis of their estimated size and firmness, it was possible to show that the histopathological assessment of only four to six nodes furnishes a reasonably acceptable alternative evaluation of qualitative axillary node status. This appears to compare favourably with mathematical models (considering all randomly selected nodes as equivalent), which state that at least 10 to 11 nodes should be examined for a reliably negative (accuracy 90\% or above) qualitative axillary nodal status..$^{22}$ This latter requirement does not take into account qualitative features of the lymph nodes such as size, consistency, or localisation, and this has resulted in a predominantly mathematical approach to what is a complex biological problem. Localisation is also neglected in this model which examines the 3-6 most obvious nodes, but the other features are not neglected. Such a sampling approach could result in a reduction in the minimum number of lymph nodes required for reliable staging as node negative disease without diminishing sensitivity.

The results reported here raise several issues. First, they provide further evidence of the reliability of taking out the four to six most obvious nodes during axillary surgery for breast cancer. Although this study did not test the ability of the surgeons to identify the most obvious nodes, it is presumed that the nodes identified as the largest ones in this study are identified with high confidence by surgeons during the operations. Most surgeons who sample the axilla take nodes from level I, but a failure to find the required number of nodes at this level would usually result in a search for palpable nodes at level II, and this is why the failure to separate the analysed nodes into levels should be only a minor limitation in this study.

Second, it is also likely that the nodes estimated as being the most obvious ones in this study are also those that a pathologist would identify as such when cutting up an axillary clearance specimen. This is why node negative cancers diagnosed as such from six to seven nodes should not be considered inadequately staged because of the seemingly suboptimal number of lymph nodes assessed, since the most obvious nodes were probably examined. This conclusion also highlights the erroneous oncology practice of giving regional radiation treatment to the axilla when node negativity was established from less than 10 nodes from an axillary clearance specimen.

Third, the method of sampling the four to six most obvious nodes in the histopathology laboratory could be an alternative cost sparing approach for the evaluation of axillary clearance specimens in poorer countries. This alternative should be further tested, since most authorities would be against such a "negligent" pathology work up, and most recommend the recovery of as many nodes as possible from the axillary fat. In a previous study based on an earlier period than the one reported here, we found that more than half of our specimens had a seemingly suboptimal number of lymph nodes recovered and examined microscopically (an average of seven nodes per axillary specimen). By applying a mathematical model, we concluded that at least 10 nodes must be examined for a reliable identification of the pN0 category. ${ }^{23}$ We then audited the process of cutting up and raised the average number of lymph nodes per specimen to 22 . By applying the same mathematical model to these cases, the suggested minimum number of lymph nodes to be investigated increased to 27 , with all specimens having 10-42 nodes examined. However, the assessment of more nodes did not seem to influence the rate of detection of node positive tumours, and indicated that fewer than 10 nodes could also give a reliable histopathological staging of breast cancer. ${ }^{24}$

Clinical evidence of the truth of our first assumption comes from several studies. Lymph node sampling is a diagnostic procedure used in several countries, for example the United Kingdom and Denmark. It is a term applied to different methods of removing axillary lymph nodes for staging (prognostic) purposes. Because of the multiplicity of techniques and the meaning of "axillary sampling," published reports have been contradictory, some studies suggesting reliability, ${ }^{6-10}$ and others a high error rate. ${ }^{15-18}$ Such a high error rate may in fact result from poorly performed surgery. ${ }^{10}$ It appears that sampling and histological work up of the four to six largest/firmest lymph nodes identified by the surgeon during the operation is a reliable alternative method of staging breast cancer. $^{79}$

In view of the data supporting the value of axillary sentinel lymph node biopsy for staging, ${ }^{11-1425}$ it is important to note that the 
nodes draining the tumour usually enlarge as a consequence of reactive hyperplasia caused by preoperative diagnostic interventions (aspiration or core biopsies), and this makes them easier to palpate during sampling. This may cause an overlap with sentinel lymphadenectomy in cases without properly performed lymphatic mapping. Our preliminary data on 40 consecutive breast cancer patients undergoing sentinel lymphadenectomy before completing dissection suggest that the sentinel lymph node identified by patent blue mapping is in most cases ( $95 \%)$ included among the six largest/ firmest nodes, defined by the same way as in this study. ${ }^{25}$ We are aware of another study using gamma probe guidance for identifying the sentinel lymph nodes in which investigators found a $72 \%$ overlap between the sentinel nodes and a four node sample performed before using the gamma probe. ${ }^{26}$

In the present era, when lymphatic mapping and sentinel lymphadenectomy is apparently the best approach to staging early breast cancer, such a sampling procedure could be followed in cases where no sentinel lymph node has been identified or where there are multiple tumours, when identification of sentinel lymph nodes may be unreliable. ${ }^{27}$ This reduction in the number of nodes assessed could also make the more sensitive methods of detecting axillary metastases (serial sectioning, immunostaining) more cost-effective. Patients with positive axillary sampling could then be treated by either irradiation ${ }^{28}$ or therapeutic dissection. In this way, patients with node negative tumours could avoid axillary dissection and its possible morbidity without loss of axillary node status, which remains the most powerful predictor of prognosis.

A possible drawback of these results is that they were obtained on breast cancer cases with palpable tumours. However, the results produced by sampling in the United Kingdom indicate that this method may also be of value in cases detected by screening.

1 Fisher B, Wolmark N, Bauer M, et al. The accuracy of clinical nodal staging and of limited axillary dissection as a determinant of histologic nodal status in carcinoma of the breast. Surg Gynecol Obstet 1981;152:765-72.

2 Danforth DN, Findlay PA, McDonald HD, et al. Complete axillary lymph node dissection for stage I-II carcinoma of axillary lymph node dissection for stage

3 McLean RG, Ege GN. Prognostic value of axillary lymphoscintigraphy in breast carcinoma patients. 7 Nucl Med 1986;27:1116-24.

4 Crowe JP, Lee MD, Adler MD, et al. Positron emission tomography and breast masses: comparison with clinical, mammographic, and pathological findings. Ann Surg Oncol 994;1:132-40.

5 Tate J, Lewis V, Archer T, et al. Ultrasound detection of axillary lymph node metastases in breast cancer. Eur 7 Surg Oncol 1989;15:139-42.

6 Forrest APM, Roberts MM, Cant E, et al. Simple mastectomy and pectoral node biopsy. Br F Surg 1976;63: 569-75.

7 Kjaergaard J, Blichert-Toft M, Andersen JA, et al, and the false negative nodal staging in conjunction Probability of xillary dissection in breast cancer. Br F Surg 1985;72:3657 .

8 Steele RJC, Forrest APM, Gibson T, et al. The efficacy of lower axillary sampling in obtaining lymph node status in
breast cancer; a controlled randomised trial. Br $\mathcal{F}$ Surg breast cancer; a

9 Fisher CJ, Boyle S, Burke M, et al. Intraoperative assessment of nodal status in the selection of patients with breast cancer for axillary clearance. Br F Surg 1993;80:4578.

10 Greenall MJ. Why I favour axillary node sampling in the management of breast cancer. Eur F Surg Oncol 1995;21:2-

11 Krag DN, Weaver DL, Alex JC, et al. Surgical resection and radiolocalization of sentinel lymph node in breast cancer using a gamma probe. Surg Oncol 1993;2:335-40.

12 Giuliano AE, Kirgan DM, Guenther JM, et al. Lymphatic mapping and sentinel lymphadenectomy for breast cancer. Ann Surg 1994;220:391-401.

13 Albertini JJ, Lyman GH, Cox C, et al. Lymphatic mapping and sentinel node biopsy in the patient with breast cancer. FAMA 1996;276:1818-22.

14 Veronesi U, Paganelli G, Galimberti V, et al. Sentinel-node biopsy to avoid axillary dissection in breast cancer with clinically negative lymph-nodes. Lancet 1997;349:1864-7.

15 Davies GC, Millis RR, Hayward JL. Assessment of axillary lymph node status. Ann Surg 1980;192:148-151.

16 Kissin MW, Thompson PH, Price AB, et al. The inadequacy of axillary sampling in breast cancer. Lancet 1982;i:121012.

17 Pigott J, Nichols MD, Maddox WA, et al. Metastases to the upper levels of axillary nodes in carcinoma of the breast and its implications for nodal sampling procedures. Surg Gynecol Obstet 1984;158:255-9.

18 Mathiesen O, Carl J, Bonderup O, et al. Axillary sampling and the risk of erroneous staging of breast cancer. Acta Oncol 1990;29:721-5.

19 Kinne DW. Controversies in primary breast cancer management. Am f Surg 1993;166:502-8

20 Davidson T. Why I favour axillary node clearance in the management of breast cancer. Eur F Surg Oncol 1995;21:57.

21 Spiessl B, Beahrs OH, Hermanek P, et al, eds. Breast tumours. In: UICC TNM atlas. Berlin: Springer-Verlag, 1990:173-83.

22 Kiricuta CI, Tausch J. A mathematical model of axillary lymph node involvement based on 1446 complete axillary dissections in patients with breast carcinoma. Cancer 1992; 69:2496-501.

23 Cserni G. Changing trends in lymph node recovery from axillary clearance specimens in breast cancer: possible mplications for the quantitative axillary status from a 17 year retrospective study Eur F Oncol 1997;2:403-8.

24 Cserni G. How to improve low lymph node recovery rates from axillary clearance specimens of breast cancer? A short-term audit. $\mathcal{F}$ Clin Pathol 1998;51:846-9.

25 Cserni G. Estimating the overlap between sentinel lymph nodes and axillary node samples in breast cancer. Pathol Oncol Res 1999;5:129-33.

26 Macmillan RD, Hadjiminas D, Barbera D, et al. Sentinel node biopsy and axillary node sampling-how they compare. Eur f Surg Oncol 1998;24:618.

27 Statman R, Giuliano AE. The role of the sentinel lymph node in the management of patients with breast cancer. Adv Surg 1997;30:209-21.

28 Kingston RD, Bridger J, Smith CJ, et al. Axillary sampling and radiotherapy does not compromise the patient with early breast cancer: Trafford database experience. Eur $\mathcal{F}$ Surg Oncol 1995;21:364-7. 